\title{
Explicit Real Cubic Surfaces
}

\author{
Irene Polo-Blanco and Jaap Top
}

Abstract. The topological classification of smooth real cubic surfaces is recalled and compared to the classification in terms of the number of real lines and of real tritangent planes, as obtained by L. Schläfli in 1858. Using this, explicit examples of surfaces of every possible type are given.

\section{Introduction}

It is a well-known result of A. Clebsch, (see [B]) that every smooth cubic surface $S \subset \mathbb{P}^{3}(\mathbb{C})$ can be obtained by blowing up $\mathbb{P}^{2}(\mathbb{C})$ at six points in general position, i.e., no three are on a line and not all six lie on a conic. In other words, $S$ admits a blow-down morphism $\varphi: S \rightarrow \mathbb{P}^{2}$, characterized by the properties that $\varphi$ is an isomorphism from $S \backslash \bigcup_{i=1}^{6} \ell_{i}$ to $\mathbb{P}^{2} \backslash\left\{P_{1}, \ldots, P_{6}\right\}$, in which the $\ell_{i} \subset S$ are six pairwise disjoint lines and the $P_{i} \in \mathbb{P}^{2}$ are six pairwise different points. Furthermore, $\varphi\left(\ell_{i}\right)=P_{i}$. (It turns out that $S$ admits, up to linear automorphisms of $\mathbb{P}^{2}$, precisely 36 blow-down morphisms to $\mathbb{P}^{2}$.)

Any such surface $S$ contains precisely 27 lines, as was originally proved by G. Salmon after correspondence with A. Cayley. Another very well-known fact is the description of the 27 lines in terms of a blow-down morphism $\varphi: S \rightarrow \mathbb{P}^{2}$ : the lines are the six pre-images $\ell_{i}=\varphi^{-1}\left(P_{i}\right)$, the fifteen irreducible components different from $\ell_{i}$ and $\ell_{j}$ of the pre-images $\varphi^{-1}\left(L_{i j}\right)$, in which $L_{i j}$ denotes the line in PP $^{2}$ containing $P_{i}$ and $P_{j}$, and finally the six components different from lines $\ell_{j}$ in the pre-images $\varphi^{-1}\left(P_{i}^{\prime}\right)$ where $P_{i}^{\prime}$ is the conic in $\mathbb{P}^{2}$ containing the five points $\left\{P_{1}, \ldots, P_{6}\right\}-\left\{P_{i}\right\}$. From this description it is immediate which lines intersect and which lines do not. A modern reference for all these well-known facts on cubic surfaces is [B, Proposition IV.9, Proposition IV.12, Theorem IV.1]. Any two intersecting lines determine a plane $P$ (the unique plane containing these two lines). The intersection $S \cap P$ then consists of three pairwise intersecting lines; such a plane is classically called a tritangent plane of S. Using the description of the lines in terms of a blow-down morphism, it is very easy to verify that any smooth cubic surface $S$ has precisely 45 tritangent planes.

Within ten years after Salmon proved his result on the 27 lines, the Swiss mathematician L. Schläfli [Sch] classified smooth cubic surfaces defined over $\mathbb{R}$ according to their number of real lines and real tritangent planes.

Theorem 1.1 (Schläfli) The number of real lines and of real tritangent planes on any smooth, real cubic surface is one of the following, in which each pair really occurs: $(27,15),(15,15),(7,5),(3,7),(3,13)$.

Received by the editors October 24, 2005; revised December 9, 2005.

AMS subject classification: Primary: 14J25; secondary: 14J80, 14P25, $14 \mathrm{Q} 10$.

(C)Canadian Mathematical Society 2008. 
This result was also obtained by L. Cremona [Cr].

Given such a smooth, cubic surface $S$ over $\mathbb{R}$, an alternative classification is by the topological structure on the space of real points $S(\mathbb{R}) \subset \mathbb{P}^{3}(\mathbb{R})$. This was done by several authors including H. Knörrer and T. Miller [KM], R. Silhol [Si], and J. Kollár $[\mathrm{K}]$. The result is as follows.

Theorem 1.2 A smooth, real cubic surface is isomorphic over $\mathbb{R}$ to a surface of one of the following types.

(i) A surface $X$ obtained by blowing up $\mathbb{P}^{2}{ }^{2}$ in six real points (no three on a line, not all six on a conic). In this case, $X(\mathbb{R})$ is the non-orientable compact connected surface of Euler characteristic - 5 .

(ii) A surface X obtained by blowing up $\mathbb{P}^{\mathrm{P}^{2}}$ in four real points and a pair of complex conjugate points (again, no three on a line, not all six on a conic). In this case, $X(\mathbb{R})$ is the non-orientable compact connected surface of Euler characteristic-3.

(iii) A surface $X$ obtained by blowing up $\mathbb{P}^{2}$ in two real points and two pairs of complex conjugate points (again, no three on a line, not all six on a conic). In this case, $X(\mathbb{R})$ is the non-orientable compact connected surface of Euler characteristic -1 .

(iv) A surface $X$ obtained by blowing up $\mathrm{P}^{\mathrm{P}^{2}}$ in three pairs of complex conjugate points (again, no three on a line, not all six on a conic). In this case, $X(\mathbb{R})$ is homeomorphic to $\mathbb{P}^{2}(\mathbb{R})$, which has Euler characteristic 1 .

(v) A surface $X$ constructed as follows. Take a smooth, real conic given as $F=0$ and five real points $P_{1}, \ldots, P_{5}$ on it. Take a sixth real point $P_{6} \neq P_{5}$ on the tangent line to the conic at $P_{5}$, such that no three of the six points are on a line. Write the conic through $P_{1}, \ldots, P_{4}, P_{6}$ as $G=0$. The rational map $\mathbb{P}^{2} \ldots \rightarrow \mathbb{P}^{2}$ defined by $P \mapsto \tau(P)$, in which $\{P, \tau(P)\}$ is the intersection of the conic given by $G(P) F-F(P) G=0$ and the line through $P$ and $P_{6}$, then extends to an involution $\tau$ on the blow-up $B$ of $\mathrm{PP}^{2}$ in the points $P_{1}, \ldots, P_{6}$. Define $X$ as the quotient $B \times \operatorname{Spec}(\mathbb{C}) /(\tau \times c)$ in which $c$ is complex conjugation. Then $X(\mathbb{R})$ consists of two connected components, one homeomorphic to the 2-sphere $S^{2}$ and the other to $\mathbb{P}^{2}(\mathbb{R})$.

A straightforward and amusing consequence is the following.

Corollary 1.3 A smooth, cubic surface $S$ defined over $\mathbb{R}$ does not admit any real blowdown morphism to $\mathbb{P}^{2}$ if and only if $S(\mathbb{R})$ is not connected.

Note that the if part of this corollary is obvious for a purely topological reason: any surface admitting a real blow-down morphism to a variety whose real points form a connected space is itself connected. The corollary yields a visual way to recognize real, smooth cubic surfaces not admitting any real blow-down morphism to $\mathbb{P}^{2}$.

In this paper we show (Proposition 2.1) how the description given in Theorem 1.2 implies, and, in fact, yields the same classes of real cubic surfaces, as the classical classification of Schläfli. Although this is certainly known to many experts, we did not find it in the literature. It is hoped that this note fills this little gap, and draws some attention to a beautiful classical part of algebraic geometry. Working directly from cubic equations, and as well as starting from the description given in Theorem 1.2, we present explicit examples. 
The work reported on in this paper started when Remke Kloosterman showed us an abstract proof of the existence of a so-called real Del Pezzo surface of degree two which does not admit a real blow-down morphism to $\mathbb{P}^{2}$; see also the survey paper by Kollár $[\mathrm{K}]$. Explicit equations for all cases described in Theorem 1.2 are also presented in a recent paper by Holzer and Labs [HL, Table 2]. The argument of Kloosterman relies on the observation that the anti-canonical sheaf on a Del Pezzo of degree two realizes the surface as a double cover of $\mathbb{P}^{2}$ ramified over a quartic curve. Moreover, the irreducible components of the preimage of the bitangents of this curve are the 56 smooth rational curves with self-intersection -1 in the Del Pezzo surface [AKP, Remark 3.3]. His result now follows by constructing a situation where the action of complex conjugation on these rational curves is incompatible with the existence of a real blow-down morphism to $\mathbb{P}^{2}$.

\section{Real Lines and Real Tritangent Planes}

In this section we show that Schläfli's classification is in fact the same as the topological one.

Proposition 2.1 The enumerative classification of Schläfli presented in Theorem 1.1 yields the same five classes as the topological one given in Theorem 1.2. More precisely, in the notation of the latter theorem, the number of real lines and of real tritangent planes for each type is as follows.

\begin{tabular}{||c|c|c|c|c||}
\hline type (1) & type (2) & type (3) & type (4) & type (5) \\
\hline$(27,45)$ & $(15,15)$ & $(7,5)$ & $(3,7)$ & $(3,13)$ \\
\hline
\end{tabular}

This is achieved by directly describing the real lines and the real tritangent planes for each of the five types given in Theorem 1.2.

We start by recalling some standard notation for the lines and tritangent planes.

Notation Let $S$ be the smooth cubic surface obtained by blowing up $\mathbb{P}^{2}$ at the six points $\left\{p_{1}, \ldots, p_{6}\right\}$. Denote the set of 27 lines on $S$ as follows. The image in $S \subset \mathbb{P}^{3}$ of the exceptional line corresponding to $p_{i}$, is denoted as $i$, for $i=1, \ldots 6$. Next, $i j$ is the image in $\mathbb{P}^{3}$ of the strict transforms of the line passing through $p_{i}$ and $p_{j}$. Finally, $j^{\prime}$ will be the image in $\mathbb{P}^{3}$ of the strict transform of the conic passing through all $p_{i}$, for $i \neq j$.

A tritangent plane $P$ is completely determined by the three lines $l_{i}$ such that $P \cap S=$ $l_{1} \cup l_{2} \cup l_{3}$. Denote $P=\left\langle l_{1}, l_{2}, l_{3}\right\rangle$.

Lemma 2.2 Let $S$ be the smooth cubic surface obtained by blowing up $\mathbb{P}^{2}$ at the six points $\left\{p_{1}, \ldots, p_{6}\right\}$. Then the 45 tritangent planes of $S$ are:

(i) The 30 planes $\left\langle i, j^{\prime}, i j\right\rangle$ with $i, j \in\{1, \ldots, 6\}, i \neq j$ and

(ii) The 15 planes $\langle i j, k l, m n\rangle$, with $\{i, j, k, l, m, n\}=\{1,2,3,4,5,6\}$.

Proof In fact, the lemma provides the description of the tritangent planes in terms of a "Schläfli double six", as was already given by L. Schläfli [Sch, p. 116, 117]. We sketch the (quite obvious) proof for convenience. 
Suppose the plane $P$ contains the line $i$. Then it does not contain any line $j$ for $j \neq i$ since such lines $i, j$ do not intersect. If $P$ contains $j^{\prime}$, then for the same reason $j \neq i$. Under this condition, $i$ and $j^{\prime}$ indeed intersect, and both intersect the line $i j$. Hence $P=\left\langle i, j^{\prime}, i j\right\rangle$. It is easily seen that this is the only type of tritangent plane containing a line $i$ or a line $j^{\prime}$.

Two different lines $i j$ and $k l$ intersect precisely, when the numbers $\{i, j\}$ and $\{k, l\}$ are disjoint. If this is the case, then $m n$ (with $\{m, n\}$ the remaining two numbers in $\{1, \ldots, 6\})$ is the unique third line intersecting both $i j$ and $k l$. This yields the tritangent planes $\langle i j, k l, m n\rangle$.

We now describe the number of real lines and real tritangent planes for each of the cases described in Theorem 1.2. This is quite simple, and somewhat similar to arguments that can be found in nineteenth century papers by L. Schläfli, L. Cremona and A. Clebsch. However, surprisingly, we did not find the result in the classical literature, nor in modern texts on cubic surfaces such as the books by B. Segre [Se], by Yu. I. Manin [M] or by R. Silhol [Si].

Notation Let " " " denote complex conjugation. We will write $\bar{j}$ (and in the same manner $\bar{j}^{\prime}, \ldots$, etc.) to denote the exceptional line corresponding to the point $\bar{p}_{i}$. Note that this equals the conjugate line of the exceptional line corresponding to $p_{i}$.

We discuss the various types described in Theorem 1.2 case by case.

Type (i) The cubic surface $S$ is obtained by blowing up six real points in $\mathbb{P}^{2}$. In this case, all 27 lines and all 45 tritangent planes are real.

Type (ii) Now $S$ is obtained by blowing up four real points and one pair of complex conjugate points, i.e., the set of six points is given by $\left\{a_{1}, \overline{a_{1}}, a_{2}, a_{3}, a_{4}, a_{5}\right\}$.

(a) The real lines are:

of type $i: 2,3,4$ and 5 ;

of type $j^{\prime}: 2^{\prime}, 3^{\prime}, 4^{\prime}$ and $5^{\prime}$;

of type $(i j):\{i, j\}=\{\bar{i}, \bar{j}\} \Leftrightarrow i j \in\{1 \overline{1}, 23,24,25,34,35,45\}$.

In total, there are $4+4+7=15$ real lines.

(b) The real tritangent planes are:

of type $\left\langle i, j^{\prime}, i j\right\rangle:$ all 12 planes with $i, j \in\{2,3,4,5\}$;

of type $\langle i j, k l, m n\rangle$ : the planes $\langle 1 \overline{1}, 23,45\rangle,\langle 1 \overline{1}, 24,35\rangle,\langle 1 \overline{1}, 25,34\rangle$.

In total, there are $12+3=15$ real tritangent planes.

Type (iii) Here $S$ is obtained by blowing up two real points and two pairs of complex conjugate points, i.e., the set of six points is given by: $\left\{a_{1}, \overline{a_{1}}, a_{2}, \overline{a_{2}}, a_{3}, a_{4}\right\}$.

(a) The real lines are:

of the kind $i: 3$ and 4 ;

of the kind $j^{\prime}: 3^{\prime}$ and $4^{\prime}$;

of the kind $(i j): 1 \overline{1}, 2 \overline{2}, 34$.

In total, there are 7 real lines. 
(b) The real tritangent planes are:

of type $\left\langle i, j^{\prime}, i j\right\rangle:\left\langle 3,4^{\prime}, 34\right\rangle$ and $\left\langle 4,3^{\prime}, 43\right\rangle$;

of type $\langle i j, k l, m n\rangle:\langle 1 \overline{1}, 2 \overline{2}, 34\rangle,\langle 34,1 \overline{2}, \overline{1} 2\rangle$ and $\langle 34,12, \overline{1} \overline{2}\rangle$.

In total, there are 5 real tritangent planes.

Type (iv) In this case, the cubic surface $S$ is obtained by blowing up three pairs of complex conjugate points. Write the points as $\left\{a_{1}, \overline{a_{1}}, a_{2}, \overline{a_{2}}, a_{3}, \overline{a_{3}}\right\}$.

(a) The only real lines in this case are $1 \overline{1}, 2 \overline{2}, 3 \overline{3}$. Hence there are precisely three real lines in $S$.

(b) We now calculate the number of real tritangent planes of $S$. Clearly, no tritangent plane of the form $\left\langle i, j^{\prime}, i j\right\rangle$ is fixed by complex conjugation. Furthermore, every real tritangent plane must contain a real line, i.e., one of the three lines $\{1 \overline{1}, 2 \overline{2}, 3 \overline{3}\}$.

The planes $\left\langle i, \bar{i}^{\prime}, i \bar{i}\right\rangle$ are not fixed by complex conjugation. The real ones are $\langle 1 \overline{1}, 2 \overline{2}, 3 \overline{3}\rangle,\langle 1 \overline{1}, 23, \overline{2} \overline{3}\rangle,\langle 1 \overline{1}, 2 \overline{3}, \overline{2} 3\rangle,\langle 2 \overline{2}, 13, \overline{1} \overline{3}\rangle,\langle 2 \overline{2}, 1 \overline{3}, \overline{1} 3\rangle,\langle 3 \overline{3}, 12, \overline{1} \overline{2}\rangle$ and $\langle 3 \overline{3}, 1 \overline{2}, \overline{1} 2\rangle$. These are the seven real tritangent planes.

Type (v) This case is obtained by changing the real structure on a special kind of surface as described in Type (i).

Let $a_{1}, \ldots, a_{6}$ be the six real points in $\mathbb{P}^{2} 2$ which will be blown up. By construction, the line connecting $a_{5}$ and $a_{6}$ is tangent in $a_{5}$ to the conic passing through $a_{1}, \ldots, a_{5}$. Let $B$ be the real cubic surface in $\mathrm{P}^{3}$ obtained by the usual embedding of the blow-up of $\mathrm{P}^{2}$ in the points $a_{i}$. We first describe the action of the involution $\tau$ on the 27 lines in $B$.

First, let $\{1,2,3,4\}=\{i, j\} \cup\{k, l\}$. Then $\tau: i j \leftrightarrow k l$. Indeed, take a general point $u$ on the line $L_{i j}$ containing $a_{i}$ and $a_{j}$. The (singular) conic through $a_{1}, \ldots, a_{4}, u$ equals the union $L_{i j} \cup L_{k l}$, and since $\{u, \tau(u)\}=\left(L_{i j} \cup L_{k l}\right) \cap L$ where $L$ is the line connecting $u$ and $a_{6}$, the image $\tau(u)$ is a general point on $L_{k l}$. In a similar way, using the conic through $a_{1}, \ldots, a_{5}$ and observing that $L_{56}$ is tangent to this conic, it follows that $\tau: 5 \leftrightarrow 5$ and $\tau: 6^{\prime} \leftrightarrow 6^{\prime}$. Now take a general point $u$ on the line $L_{56}$. Clearly, its image under $\tau$ is again a general point on $L_{56}$, hence $\tau: 56 \leftrightarrow 56$.

Next, take $j \in\{1,2,3,4\}$ and consider a general point $u \in L_{j 6}$. The conic $C_{u}$ passing through $a_{1}, \ldots, a_{4}, u$ then obviously intersects $L_{j 6}$ in the points $u$ and $a_{j}$. This means $\tau(u)=a_{j}$, so in particular, the rational involution map $\tau$ on $\mathrm{P}^{2}$ restricted to $L_{j 6}$ is not even bijective. However, changing the point $u$ also changes the direction of the tangent line to $C_{u}$ at $a_{j}$. On the blow up $B$, this shows $\tau: j 6 \leftrightarrow j$. A similar argument yields that $\tau$ maps the general point on the conic through $a_{1}, \ldots, a_{4}, a_{6}$, to $a_{6}$, and $\tau: 5^{\prime} \leftrightarrow 6$.

It is now a straighforward computation to determine the action of $\tau$ on the remaining lines of $B$ : one may use the observation that for any pair of lines $\ell_{1}, \ell_{2} \subset B$, the intersection number $\ell_{1} \cdot \ell_{2}$ equals $\tau\left(\ell_{1}\right) \cdot \tau\left(\ell_{2}\right)$. Using this, it follows that for each $j \in\{1,2,3,4\}$ one has $\tau: j^{\prime} \leftrightarrow j 5$.

With this information one can also describe the action of complex conjugation $c$ on the real cubic surface $S=B \times \operatorname{Spec}(\mathbb{C}) /(\tau \times c)$. Namely, over $\mathbb{C}$ this surface is isomorphic to $B$, giving an identification of the lines and tritangent planes of $B$ with 
those of $S$. Then by construction, a line/tritangent plane of $S$ is real if and only if the corresponding line/tritangent plane of $B$ is fixed by $\tau \times c$. Since $c$ fixes all lines and tritangent planes of $B$, this means we must count the ones fixed by $\tau$.

(a) As is explained above, $5,6^{\prime}$ and 56 are the only lines in $B$ fixed by $\tau$. Hence $S$ contains precisely three real lines.

(b) To determine the tritangent planes fixed by $\tau$, observe that any such plane contains at least 1 line in $B$ fixed by $\tau$, so at least one of the lines $5,6^{\prime}$ or 56 .

The tritangent planes fixed by $\tau$ then are:

of type $\left\langle i, j^{\prime}, i j\right\rangle$ : the 4 planes $\left\langle 5, j^{\prime}, j 5\right\rangle$ with $j \in\{1,2,3,4\}$ and the 4 planes $\left\langle i, 6^{\prime}, i 6\right\rangle$ with $i \in\{1,2,3,4\}$ and the 2 planes $\left\langle 5,6^{\prime}, 56\right\rangle$ and $\left\langle 6,5^{\prime}, 56\right\rangle$;

of type $\langle i j, k l, m n\rangle$ : the three planes $\langle i j, k l, 56\rangle$ with $\{i, j, k, l\}=\{1,2,3,4\}$.

This gives 13 planes, hence $S$ contains precisely 13 real tritangent planes. Note that in exactly one of them, namely in the one corresponding to $\left\langle 5,6^{\prime}, 56\right\rangle$, the three lines in $S$ contained in it are all real as well.

Remark In particular, the above description shows that every smooth, real cubic surface has at least one real tritangent plane that contains three real lines of the surface. In the case that the total number of real lines equals three, this means that these three lines pairwise intersect and that in fact this real tritangent plane is unique. This is an observation already made by Schläfli [Sch, p. 118 cases D, E].

\section{Examples}

Example 3.1 Let $S_{\lambda}$ be the cubic surface in $\mathbb{P}^{4}$ given by the equations:

$$
\lambda x^{3}+y^{3}+z^{3}+w^{3}+t^{3}=0, \quad x+y+z+w+t=0 .
$$

An equation for $S_{\lambda}$ in $\mathbb{P}^{3}$ is $\lambda x^{3}+y^{3}+z^{3}+w^{3}+(-x-y-z-w)^{3}=0$. A straightforward calculation shows that $S_{\lambda}$ is a smooth cubic surface for all $\lambda \in \mathbb{C}$, except $\lambda=1 / 4$ and $\lambda=1 / 16$. In case $\lambda=1 / 16$, the unique singular point is $(x: y: z: w: t)=(-4: 1: 1: 1: 1)$. For $\lambda=1 / 4$, there are exactly four singular points, given by taking $x=-2$, three of the remaining coordinates +1 , and the last one -1 . In fact, the number of isolated singularities on a cubic surface cannot exceed four, and the surface with four such singular points is unique. This surface was first studied by A. Cayley and it is called the Cayley cubic surface; $c f$. [Hu, pp. 115-122]. Some nineteenth century plaster models of it appear in the celebrated Rodenberg series; see [F, pp. 16-17], [Po2, Serie VII, nr. 2-6].

For real $\lambda>1 / 4$, all $S_{\lambda}(\mathbb{R})$ are topologically the same. The special one with $\lambda=1$ was first studied in 1871 by A. Clebsch [Cl, $\S 16]$. He showed that all 27 lines on this surface are real (see also [Hu, pp. 122-128]), hence for $\lambda>1 / 4$, the surface $S_{\lambda}$ is of Type (i). An explicit blow-down morphism, defined over $(\mathbb{Q}$, in the case $\lambda=1$ was constructed by one of the authors [Po2, pp. 52-56].

Similarly, for all real $\lambda<1 / 16$, the $S_{\lambda}(\mathbb{R})$ are homeomorphic. The case $\lambda=0$ yields the Fermat cubic surface. It is a well-know exercise [Ha, Exc. V 4.16], to show that it contains exactly three real lines and seven real tritangent planes. Hence for $\lambda<1 / 16$, the surface $S_{\lambda}$ is of Type (iv) (see [Se] for an intuitive proof of this). An 
explicit blow-down morphism, defined over $(\mathbb{O}$, in the case $\lambda=0$ was presented by N. Elkies [El] and also by one of us, [Po2, pp. 59-62].

In the singular surface $S=S_{1 / 16}$, the point $(-4,1,1,1,1)$ is an isolated point of the real locus $S(\mathbb{R})$. To see this, use the equation

$$
x^{3} / 16+y^{3}+z^{3}+1-(x+y+z+1)^{3}=0
$$

for an open affine part of $S$; on this part, we consider the point $(-4,1,1)$. The change of variables $r=x+4, s=y-1, t=z-1$ yields the new equation

$$
6 t^{2}+6 s^{2}+\frac{9}{4} r^{2}+6 r s+6 s t+6 r t+F(r, s, t)
$$

in which $F$ is a homogeneous cubic. Clearly, the quadratic part of this defines a positive definite form, hence $(0,0,0)$ is an isolated point in the real locus defined by the equation.

In fact, what happens in $S_{\lambda}(\mathbb{R})$ when $\lambda$ decreases from $1 / 4$ to $1 / 16$ is that for $\lambda=1 / 4$ we have a 2 -sphere which is connected to the rest of the surface in the four singular points. When $\lambda$ decreases, the contact of this 2 -sphere with the rest of the surface disappears. The 2 -sphere becomes smaller until at $\lambda=1 / 16$ it shrinks to a single point (the singular point), and for $\lambda<1 / 16$ this point has disappeared. In particular, what we claim here is that for $1 / 16<\lambda<1 / 4$, the real locus $S_{\lambda}(\mathbb{R})$ is not connected and hence $S_{\lambda}$ is of Type (v). This can be shown topologically. Instead, we briefly sketch below how one finds the three real lines and 13 real tritangent planes in the case $1 / 16<\lambda<1 / 4$.

Observe that the three real lines given in parametric equations as

$$
l_{1}=(0:-t: t: 1), \quad l_{2}=(0:-1: t: 1), \quad l_{3}=(0: t:-1: 1)
$$

are contained in $S_{\lambda}$.

Let $H$ be a plane containing $l_{1}$. One can write $H=\{a x+b(y+z)=0\}$. Suppose first that $a=0$. This describes the real tritangent plane $H=\{y+z=0\}$, meeting $S$ in $l_{1}$ and in a pair of complex conjugate lines. In case $a \neq 0$, the plane can be given as $H_{b}=\{x+b y+b z=0\}$. We count the number of tritangent planes of this type.

One has $H_{b} \cap S_{\lambda}=l_{1} \cup C_{b}$ where $C_{b}$ is a (possibly reducible) conic. In order for $H_{b}$ to be a tritangent plane, we need $C_{b}$ to be singular. Since $1 / 16<\lambda<1 / 4$, this happens for exactly four different real values of $b$, which gives us four real tritangent planes (in particular, the case $b=0$ yields $H=\{x=0\}$, which is the plane that contains the three real lines $l_{1}, l_{2}$ and $l_{3}$ ). We have then counted five real tritangent planes. Again using $1 / 16<\lambda<1 / 4$, it follows that each of them, apart from $\{x=0\}$, contains $l_{1}$ and a pair of complex conjugate lines. By proceeding in the same way with the tritangent planes containing $l_{2}$ and $l_{3}$ respectively, we find four real tritangent planes for each case, all containing $l_{2}$, resp. $l_{3}$, as the only real line (apart from the plane $H=\{x=0\}$ that contains all of $l_{1}, l_{2}$ and $l_{3}$ ). In total, we have now counted 12 pairs of complex conjugate lines and three real lines, hence these are all the lines of $S_{\lambda}$. Since every real tritangent plane contains a real line of $S_{\lambda}$, the above calculation in fact yields all real tritangent planes of $S_{\lambda}$. Therefore, the surface contains precisely $5+4+4=13$ real tritangent planes.

This finishes the calculation. 
Example 3.2 Instead of starting from cubic equations directly, one can of course derive such equations starting from six points in the plane. A very convenient way to do this is described by T. Shioda [Sh, $\S 6]$. His observation is that, although the six points are required not to be contained in any conic, they will certainly be smooth points on some irreducible, cuspidal cubic curve. After a linear transformation, one may assume that this cubic curve $C$ is given by the equation $y^{2} z-x^{3}=0$. Then

$$
t \mapsto a_{t}:=\left(t: 1: t^{3}\right)
$$

defines an isomorphism of algebraic groups over $(0)$ from the additive group $\left(\mathrm{Gr}_{a}\right.$ to the group of smooth points on $C$ (in which $(0: 1: 0)$ is taken as the neutral element). The latter group has the property that three points $a_{t_{1}}, a_{t_{2}}, a_{t_{3}}$ in it, are on a line precisely when $t_{1}+t_{2}+t_{3}=0$ and similarly, six smooth points $a_{t_{j}}$ are on a conic if and only if $\sum t_{j}=0$.

Now, given six values $t_{j} \in \mathbb{G}_{a}$ which are pairwise different such that no three of them add to 0 and $\sum t_{j} \neq 0$, Shioda [Sh, Thm. 14] explicitly gives an equation for the corresponding smooth, cubic surface. We give some examples of this.

Take four values $t_{1}, \ldots, t_{4}$ and put $t_{5}:=-\left(t_{1}+t_{2}+t_{3}+t_{4}\right) / 2$ and $t_{6}:=t_{1}+$ $t_{2}+t_{3}+t_{4}$. Assume that the $t_{j}$ are pairwise different, no three of them add to 0 and $\sum t_{j} \neq 0$. With this choice, the conic through $a_{t_{1}}, \ldots, a_{t_{5}}$ meets $C$ in $a_{t_{5}}$ with multiplicity two ( since $t_{1}+t_{2}+t_{3}+t_{4}+2 \cdot t_{5}=0$ ). The tangent line to this conic at $a_{t_{5}}$ is by construction tangent to $C$ as well, and because $t_{6}+t_{5}+t_{5}=0$, the third point of intersection with $C$ is $a_{t_{6}}$. Hence this is a configuration as is used in the construction of cubic surfaces of Type (v). As an explicit example, take $\left(t_{1}, \ldots, t_{6}\right):=$ $(0,1,2,5,-4,8)$. The corresponding cubic surface of Type (i) has equation

$$
y^{2} w+2 y z^{2}=x^{3}-\frac{964825}{768} x w^{2}-\frac{79}{2} x z^{2}+\frac{433748125}{55296} w^{3}+\frac{141859}{96} z^{2} w
$$

The involution $\tau$ is in this case given as $\tau(x: y: z: w):=(x: y:-z: w)$. This implies that after changing the sign of the coefficients of $y z^{2}$ and $x z^{2}$ and $z^{2} w$, a cubic surface of Type (v) is obtained.

Starting from the set $\{ \pm \sqrt{-1}, 1 \pm \sqrt{-1}, \pm 1\}$, the method yields the cubic surface of Type (iii), given by

$y^{2} w+2 y z^{2}=x^{3}-\frac{3025}{8748} x w^{2}+\frac{55}{81} x z w-\frac{1}{3} x z^{2}-\frac{5525}{354294} w^{3}+\frac{8345}{39366} z w^{2}+\frac{67}{243} z^{2} w$

To obtain an example of Type (ii), one takes four real values and one pair of complex conjugates. For example, $\{ \pm 1, \pm \sqrt{-1}, 2,3\}$ yields the equation

$$
\begin{aligned}
y^{2} w+2 y z^{2}=x^{3}-\frac{138025}{34992} x w^{2}-\frac{245}{81} & x z w-\frac{16}{3} x z^{2} \\
+ & \frac{48982975}{5668704} w^{3}+\frac{600565}{78732} z w^{2}+\frac{1439}{486} z^{2} w .
\end{aligned}
$$


Example 3.3 As a final remark, an alternative way to produce examples of real smooth cubic surfaces with exactly three real lines and 13 real tritangent planes (i.e., of Type (v)), is by changing the real structure on certain surfaces. For example, start from the Clebsch diagonal cubic surface given by $x^{3}+y^{3}+z^{3}+w^{3}-(x+y+z+w)^{3}=0$. This is a surface of Type (i). The change of coordinates $x_{0}=x+y, x_{1}=(x-y) \sqrt{-1}$, $x_{2}=z$, and $x_{3}=w$ leads to the equation $2 x_{0}^{3}-3 x_{0} x_{1}^{2}+8 x_{2}^{3}+8 x_{3}^{3}-8\left(x_{0}+x_{2}+x_{3}\right)^{3}=0$. This corresponds to a cubic surface containing exactly three real lines and 13 real tritangent planes, hence of Type (v). More details concerning this method may be found in [Po2], in particular Proposition 6 and the proof of Proposition 4.

\section{References}

[AKP] A. Artebani, R. Kloosterman, and M. Pacini, A new model for the theta divisor of the cubic threefold. Le Matematiche, 58(2003), no. 2, 201-236.

[B] A. Beauville, Complex Algebraic Surfaces. London Mathematical Society Lecture Note Series 68. Cambridge University Press, Cambridge, 1983.

[Cl] A. Clebsch, Ueber die Anwendung der quadratischen Substitution auf die Gleichungen 5ten Grades und die geometrische Theorie des ebenen Fünfseits. Math. Ann. 4(1871), no. 2, 284-345.

[Cr] L. Cremona, Mémoire de géométrie pure sur les surfaces du troisiéme ordre, J. Math Pures Appl. 68(1868), 1-133.

[El] N. Elkies, Complete cubic parametrization of the Fermat cubic surface $w^{3}+x^{3}+y^{3}+z^{3}=0$. http://www.math.harvard.edu/ elkies/4cubes.html

[F] G. Fischer, ed. Mathematische Modelle, Vieweg, Braunschweig, 1986.

[Ha] R. Hartshorne, Algebraic Geometry. Graduate Texts in Mathematics 52. Springer-Verlag, New York, 1977.

[HL] S. Holzer, and O. Labs, Illustrating the classification of real cubic surfaces. In: Algebraic Geometry and Geometric Modeling. Springer, Berlin, 2006, pp. 119-134.

[Hu] B. Hunt, The Geometry of some special Arithmetic Quotients, Lecture Notes in Mathematics 1637, Springer-Verlag, Berlin, 1996.

[KM] H. Knörrer, and T. Miller, Topologische Typen reeller kubischer Flächen, Math. Z. 195(1987), no. 1, 51-67.

[K] J. Kollár, Real algebraic surfaces, arXiv:AG/9712003, 1997.

[M] Y. I. Manin, Cubic Forms. Second edition. North-Holland, 1974, 1986.

[Po1] I. Polo-Blanco, Mathematical models of surfaces. Inventory, Univ. of Groningen, 2004 http://www.math.rug.nl/models

[Po2] I. Polo-Blanco, Theory and History of Geometric Models. Ph.D. thesis, Groningen, 2007. http://dissertations.ub.rug.nl/faculties/science/2007/i.polo.blanco/.

[Sch] L. Schläfli, An attempt to determine the twenty-seven lines upon a surface of the third order, and to divide such surfaces into species in reference to the reality of the lines upon the surface, Quart. J. Pure Appl. Math. 2 (1858), 110-120.

[Se] B. Segre, The Non-Singular Cubic Surfaces. Oxford, Oxford University Press, 1942.

[Sh] T.Shioda, Weierstrass transformations and cubic surfaces. Comment. Math. Univ. St. Paul. 44(1995), no. 1, 109-128.

[Si] R. Silhol, Real Algebraic Surfaces, Lecture Notes in Mathematics 1392, Springer-Verlag, Berlin, 1989.

IwI-RuG

P.O. Box 800

9700 AV Groningen

The Netherlands

e-mail: j.top@rug.nl 